\title{
GEOMETRICALLY NON-LINEAR FREE IN-PLANE VIBRATION OF FUNCTIONALLY GRADED CIRCULAR ARCH WITH UNIFORM CROSS-SECTION
}

\author{
O. Outassafte ${ }^{1}$, A. Adri ${ }^{1}$, Y. El khouddar ${ }^{1}$, S. Rifai ${ }^{1}$ and R. Benamar ${ }^{2}$ \\ ${ }^{1}$ Laboratoire de Mécanique Productique et Génie Industriel, Ecole Supérieure de Technologie \\ Hassan II University of Casablanca, B.P.8012, Oasis, Casablanca, Maroc \\ Said57.rifai@gmail.com \\ ${ }^{2}$ Mohammed V University in Rabat, EMI-Rabat, LERSIM \\ B.P.765 Agdal, Rabat, Morocco. \\ rhali.benamar@gmail.com
}

e-mail:omar.outassafte@ensem.ac.ma,ahmedadri@gmail.com,yassine.elkhouddar@ensem.ac.ma

\begin{abstract}
In this present work the geometrical non-linearity in free in-plane vibration of inextensible functionally graded circular arch with uniform cross-section and pinned-pinned at both ends has been studied. For simplification, the complicating effects such as rotary inertia and shear deformation will be ignored. The sixth order differential partial equation of motion has been obtained after the inextensibility assumption. This study is based on Euler Bernoulli theory and Von Karman's assumptions. The kinetic and total strain energies due to the axial strain and bending have been discretized into a series of a finite spatial functions and derived by applying the Hamilton's principle energy. The non-linear algebraic equations were obtained and solved numerically using an approximate explicit method developed previously the so-called second formulation. A numerical results have been obtained to examine the effects of the volume fraction index on non-linear behavior of the arch. Comparison is made with the available results for the case of isotropic homogeneous circular arches: good agreement is obtained.
\end{abstract}

Keywords: geometrical non-linearity, free in-plane vibration, functionally graded circular arch, uniform cross-section, second formulation.

ISSN:2623-3347 C 2021 The Authors. Published by Eccomas Proceedia.

Peer-review under responsibility of the organizing committee of COMPDYN 2021.

doi: $10.7712 / 120121.8858 .18478$ 


\section{INTRODUCTION}

Arches have become one of the very important structures elements that are used in many areas such as aeronautics, civil engineering and mechanical engineering due to its ability to transfer loads through the combined action of bending and stretching for in-plane deformations. The equations of free in-plane vibration of an arch are very complexe when the complicating effects such as axial extension, shear deformation and rotatory inertia are taken into account. If these effects are neglected, then the equations become much simpler, but the main simplification arises if the arch axis is assumed to be also inextensible.

H. Saffari et al [1] used a finite element technique to obtain the natural frequencies and mode shapes of circular arch in free vibration analyzis. The Rayleigh-Ritz method was employed by laura et al to find the fundamental frequency of with various types of cross-section using polynomial functions as a trial function of displacement [2]. G.R. Liu, T.Y. Wu were applied $\mathrm{y}$ the generalized differential quadrature rule to investigate the free in-plane vibration of inextensible Euler-Bernoulli arches with uniform, continuously varying, and stepped crosssections. A semi analytical solution for in-plane vibration of circular arch was used by $\mathrm{A}$. Babahammou and R. Benamar [3], [4] to find the linear frequencies and mode shape of arches with various boundary conditions. The results shown a very good agreement with those available in the literature. Recently, the geometrically non-linear in free In-plane vibration of circular arches elastically restrained against rotation at the two end was investigated of $O$. Omar et al [5].

Functionally graded material can be defined as a new class of non-homogeneous composite materials made from combination of two different materials. In recent years, a lot of research has been done to study the geometrical non-linearity of the FGM and composite structure based on Benamar's method such as [6]-[10]

The free in-plane vibration of arches made of homogeneous, composite, and FG materials was the interest of numerous researchers such as . Hadi Babaei et al [11] have investigated the large amplitude free vibration of curved beam (shallow arch) resting on elastic foundation. The natural frequencies of a deep circular arch made of FG materials with arbitrary thickness and boundary conditions, have been obtained in [12]. The influence of the temperature, boundary conditions and the material graded index as well as the different geometrical parameters such as the thickness-to-mean radius ratio and the opening angle on the frequency parameters of the FG arches have been investigated by [13]. M. Javan et al [14] have studied a large amplitude thermally induced vibration analysis is investigated for shallow FGM arches under different cases of rapid heating on the top and bottom surfaces. In [15] an analytical solution to study the non-linear thermal bending and buckling of the through-the-thickness FGM shallow arches is presented, taking into account the variation of critical buckling temperature in terms of power law index, geometric parameter and opening angle.

This current work deals with the study of the geometrical non-linearity in free in-plane vibration of a FGM circular arch with uniform cross-section and pinned-pinned at both ends. This theoretical model is based on the Euler-Bernoulli theory and the Von Karman assumptions. The complicating effects such as rotary inertia and shear deformation will be ignored. The kinetic and total strain energies due to the axial strain and bending were discretized into a series of a finite spatial functions and derived using Hamilton's principle energy. Then, the non-linear algebaraic equations are obtained and solved numerically using an explicit method developed previously, the so-called second formulation. A numerical results have been obtained to 
examine the effects of the volume fraction index on the non-linear behavior of the FGM circular arch.

\section{GENERAL FORMULATION}

\subsection{Non-linear vibration}

A functionally graded circular arch made from a mixture of ceramic-metal material with a constant thicknesses is considered in this study. The effective material properties of the FGM circular arch such as Young's modulus $E(z)$, and mass density $\rho(z)$, with volume fraction index $p$ are assumed to vary continuously through the thickness of the beam, according to power law form are shown in Fig.1.

The effective of material properties $\mathrm{P}$, can be expressed as:

$$
P=P_{m} V_{m}+P_{c} V_{c}
$$

Where $P_{m}, V_{m} P_{c}$, and $V_{c}$ are the material properties and the volume fraction of the metal and ceramic respectively.

In which $V_{m}$ and $V_{c}$ are the metal and ceramic volume fractions respectively related by:

$$
V_{m}+V_{c}=1
$$

Assuming the power law distribution, the volume fraction can be written with the relation:

$$
V_{f}(z)=\left(\frac{z}{h}+\frac{1}{2}\right)^{p}
$$

Where $p$ is the volume fraction index, $0 \leq p \leq \infty$. When $\mathrm{p}$ is set to zero, the FG arch becomes fully ceramic, when $p$ is set to infinite, the FG arch becomes fully metal .
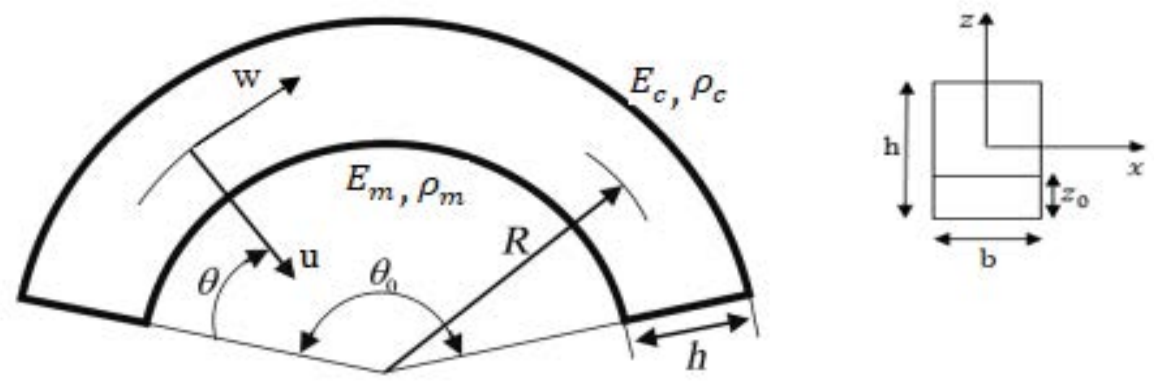

Fig 1: Schematic of a FGM circular arch

According to the power law, the young's modulus $E(z)$ and mass density are $\rho(z)$ expressed as:

$$
\begin{aligned}
& E(z)=E_{m}+\left(E_{c}-E_{m}\right) V_{f}(z) \\
& \rho(z)=\rho_{m}+\left(\rho_{c}-\rho_{m}\right) V_{f}(z)
\end{aligned}
$$


Where $\left(E_{m}, \rho_{m}\right)$ are the material properties for metal and $\left(E_{c}, \rho_{c}\right)$ are the material properties for ceramic respectively. The physical parameters of ceramic and metal are presented in Table 1.

Table 1 :The physical parameters of ceramic and metal

\begin{tabular}{lcc}
\hline Materials & $\begin{array}{c}\text { Young's modulus } \\
E / \mathrm{Gpa}\end{array}$ & $\begin{array}{c}\text { Density } \\
\rho /\left(\mathrm{kg}^{-3} \mathrm{~m}^{-3}\right)\end{array}$ \\
\hline Ceramic & 380 & 3980 \\
\hline Metal & 206 & 7800 \\
\hline
\end{tabular}

The variation the density and Young's modulus along the height of the cross-section is shown in Fig.2, by taking into account several values of the the volume fraction index.
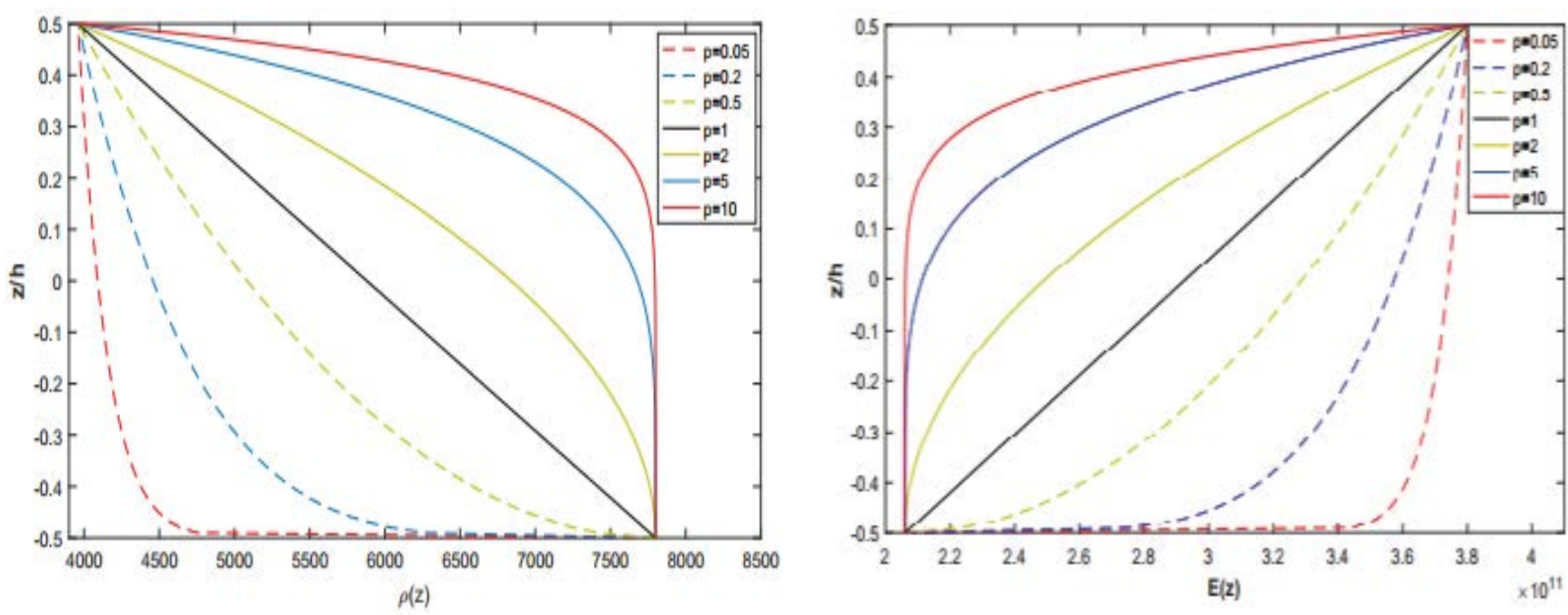

Fig. 2. Variation of the density and Young's modulus through the arch thickness for different values of gradient index p.

In this present study, the complicated effects such as shear deformation and rotary inertia are neglected because the arch is supposed to be thin. The arch axis is supposed to inextensible, the radial displacement $u$ and tangential displacement $w$ are related by [16]:

$$
\frac{\partial w}{\partial \varphi}=u
$$

The sixth order differential equation of motion may be written as:

$$
\frac{\partial^{6} w}{\partial \varphi^{6}}+2 \frac{\partial^{4} w}{\partial \varphi^{4}}+\frac{\partial^{2} w}{\partial \varphi^{2}}+\lambda^{2} \frac{\partial^{4} w}{\partial t^{2} \partial \varphi^{4}}=0
$$

$\lambda_{i}^{2}=\frac{\omega_{i}^{2} R_{i}^{* 4} \int_{-h / 2}^{h / 2} \rho d y}{\int_{-h / 2}^{h / 2} E y^{* 2} d y}$ is the non-dimensional frequency. 


$$
\begin{gathered}
z_{0}=\frac{p\left(E_{m}-E_{c}\right)}{2(p+2)\left(p E_{c}+E_{m}\right)} h \\
z^{*}=z+z_{0} \\
R^{*}=R+z_{0}
\end{gathered}
$$

$z_{0}$ denoted the height of the metal material, $z$ denoted the neutral axis of isotropic homogenous circular arch, $z^{*}$ denoted the neutral axis of the FGM circular arch, of the Where $\mathrm{R}$ denoted the radius of curvature of the geometric median of the arch and $\mathrm{R}^{*}$ denoted the radius of curvature of the neutral layer.

Considering the arch in a harmonic motion, we put:

$$
w(\varphi, t)=W(\varphi) \cos (\omega t)
$$

When the form of solution is:

$$
W(\varphi)=e^{\Omega \varphi}
$$

The characteristic equation may be written as:

$$
\Omega^{6}+2 \Omega^{4}+\left(1-\lambda^{2}\right) \Omega^{2}=0
$$

The general solution of the differential equation (7) in term of tangential displacement can be written as:

$$
W_{i}(\varphi)=C_{1} \sin (\alpha \varphi)+C_{2} \cos (\alpha \varphi)+C_{3} \sinh (\alpha \varphi)+C_{4} \cosh (\alpha \varphi)+C_{5}(\varphi)+C_{6} \quad \text { with } \quad i=1, \ldots, n
$$

Where: $\alpha=\sqrt{\lambda-1}, \beta=\sqrt{\lambda+1}$ and $\lambda \geq 1$.

The pinned-pinned boundary conditions of circular arch in term of tangential displacement may be written as:

$$
W_{i}(\varphi=0)=W_{i}\left(\varphi=\varphi_{0}\right)=\left.0 \quad \frac{d W_{i}}{d \varphi}\right|_{\varphi=0}=\left.\frac{d W_{i}}{d \varphi}\right|_{\varphi=\varphi_{0}}=\left.0 \quad \frac{d^{3} W_{i}}{d \varphi^{3}}\right|_{\varphi=0}=\left.\frac{d^{3} W_{i}}{d \varphi^{3}}\right|_{\varphi=\varphi_{0}}=0
$$

The use of the end conditions and the transfer matrix method leads to a homogeneous system. The determinant of the latest must be set equal to zero to obtain the natural frequencies, determined iteratively by the Newton-Raphson algorithm. 


\subsection{Non-linear vibration}

The kinetic energy $\mathrm{T}$ and total strain energy $V$ of the system presented in Fig. 1 may be written as a sum of the axial energy $V_{a}$ and the strain energy due to the bending $V_{b}$. The expression of $T, V_{a}$ and $V_{b}$ are expressed as:

$$
\begin{gathered}
T=\frac{R^{*}}{2} \int_{0}^{\theta_{0}}\left[b\left(\frac{\partial w_{i}^{(1)}}{\partial t}\right)^{2}+\left(\frac{\partial w_{i}}{\partial t}\right)^{2} \int_{-h / 2}^{h / 2} \rho d y\right] d t \\
V_{a}=\frac{1}{2 R^{* 3}} \int_{0}^{\theta_{0}}\left[b\left(\frac{\partial w_{i}}{\partial \theta}+\frac{\partial^{3} w_{i}}{\partial \theta^{3}}\right)^{2} \int_{-h / 2}^{h / 2} E y^{* 2} d y\right] d \theta \\
V_{b}=\frac{1}{8 R^{* 3}} \int_{0}^{\theta_{0}}\left[b\left(w_{i}+\frac{\partial^{2} w_{i}}{\partial \theta^{2}}\right)^{4} \int_{-h / 2}^{h / 2} E d y\right] d \theta \\
V=V_{a}+V_{b}
\end{gathered}
$$

Assuming harmonic, the tangential displacement function is expanded in the form of finite basic spatial functions:

$$
w_{i}(\varphi, t)=a_{i} W_{i} \sin (\omega t)
$$

By replacing $w_{i}$ in the expressions for the energies $T, V_{a}$ and $V_{b}$ can be written in the form:

$$
\begin{gathered}
T=\frac{1}{2} a_{i} a_{j} \omega^{2} m_{i j} \cos ^{2}(\omega t) \\
V_{a}=\frac{1}{2} a_{i} a_{j} k_{i j} \sin ^{2}(\omega t) \\
V_{b}=\frac{1}{2} a_{i} a_{j} a_{k} a_{l} b_{i j k l} \sin ^{4}(\omega t)
\end{gathered}
$$

The parameters $m_{i j}, k_{i j}, b_{i j k l}$ are the mass tensor, linear and the quadratic non-linear rigidity tensor of the arch in which their expressions are defined as:

$$
\begin{gathered}
m_{i j}=R^{*} \int_{0}^{\theta_{0}} b\left(\frac{\partial W_{i}}{\partial \theta} \frac{\partial W_{j}}{\partial \theta}+W_{i} W_{j}\right)_{-h / 2}^{h / 2} \rho d y d \theta \\
k_{i j}=\frac{1}{R^{* 3}} \int_{0}^{\theta_{0}} b\left[\left(\frac{\partial W_{i}}{\partial \theta}+\frac{\partial^{3} W_{i}}{\partial \theta^{3}}\right)\left(\frac{\partial W_{j}}{\partial \theta}+\frac{\partial^{3} W_{j}}{\partial \theta^{3}}\right)\right] \int_{h / 2}^{h / 2} E y^{* 2} d y d \theta \\
b_{i j k l}=\left\{\frac{1}{4 R^{* 3}} b\left[\int_{0}^{\theta_{0}}\left(W_{i}+\frac{\partial^{2} W_{i}}{\partial \theta^{2}}\right)\left(W_{j}+\frac{\partial^{2} W_{j}}{\partial \theta^{2}}\right) d \theta \int_{0}^{\theta_{0}}\left(W_{k}+\frac{\partial^{2} W_{k}}{\partial \theta^{2}}\right)\left(W_{l}+\frac{\partial^{2} W_{l}}{\partial \theta^{2}}\right)_{-h / 2}^{h / 2} E d y d \theta\right]\right\}
\end{gathered}
$$


The coefficients $a_{i}$ are unknowns as well as the frequency $\omega_{i}$. The dynamic behaviour of the conservative system may be obtained by applying Hamilton's principle as defined in equation (27) :

$$
\delta \int_{0}^{2 \pi / \omega}(V-T) d t
$$

One can obtain the following non-linear algebraic equations:

$$
\left([K]-\omega^{2}[M]\right)\{A\}+\frac{3}{2}[B(A)]\{A\}=\{0\}
$$

To obtain non-dimensional parameters, ones put:

$$
W_{i}(\varphi)=h W_{i}^{*}\left(\frac{\varphi}{\varphi_{0}}\right)=h W_{i}^{*}\left(\varphi^{*}\right) \quad \frac{m_{i j}}{m_{i j}^{*}}=b R^{*} h^{2}, \frac{k_{i j}}{k_{i j}^{*}}=\frac{b_{i j k l}}{b_{i j k l}^{*}}=\frac{b h^{2}}{R^{* 3}}
$$

Where $m_{i j}^{*}, k_{i j}^{*}$ and $b_{i j k l}^{*}$ are the non-dimensional generalized parameters given by:

$$
\begin{gathered}
m_{i j}^{*}=\int_{0}^{1}\left(\frac{1}{\theta_{0}^{2}} \frac{\partial W_{i}^{*}}{\partial \theta^{*}} \frac{\partial W_{j}^{*}}{\partial \theta^{*}}+W_{i}^{*} W_{j}^{*}\right) \theta_{0} \int_{-h / 2}^{h / 2} \rho d y d \theta^{*} \\
k_{i j}^{*}=\left\{\int_{0}^{1}\left(\frac{1}{\theta_{0}} \frac{\partial W_{i}^{*}}{\partial \theta^{*}}+\frac{1}{\theta_{0}^{3}} \frac{\partial^{3} W_{i}^{*}}{\partial \theta^{*}}\right)\left(\frac{1}{\theta_{0}} \frac{\partial W_{j}^{*}}{\partial \theta^{*}}+\frac{1}{\theta_{0}^{3}} \frac{\partial^{3} W_{j}^{*}}{\partial \theta^{3 *}}\right) \theta_{0}^{h / 2} \int_{-h / 2}^{h / 2} E y^{* 2} d y d \theta^{*}\right\} \\
b_{i j k l}{ }^{*}=\left\{\frac{h^{2}}{4} \int_{0}^{1}\left(W_{i}^{*}+\frac{\partial^{2} W_{l}^{*}}{\theta_{0}^{2} \partial \theta^{2 *}}\right)\left(W_{j}^{*}+\frac{\partial^{2} W_{j}^{*}}{\theta_{0}^{2} \partial \theta^{2 *}}\right) \theta_{0} d \theta^{*} \int_{0}^{1}\left(W_{k}^{*}+\frac{\partial^{2} W_{k}^{*}}{\theta_{0}^{2} \partial \theta^{2 *}}\right)\left(W_{l}^{*}+\frac{\partial^{2} W_{l}^{*}}{\theta_{0}^{2} \partial \theta^{2 *}}\right) \theta_{0}^{h / 2} E d y d \theta_{-h / 2}^{*}\right\}
\end{gathered}
$$

By substituting the non-dimensional parameter into equation (28) one obtain the dimensionless non-linear algebraic equations :

$$
\left(\left[K^{*}\right]-\omega^{* 2}\left[M^{*}\right]\right)\{A\}+\frac{3}{2}\left[B^{*}(A)\right]\{A\}=\{0\}
$$

$B_{i j k l}^{*}$ presents the dimensionless non-linearity tensor, $K_{i j}^{*}$ is the dimensionless rigidity matrix,$M_{i j}^{*}$ denotes the dimensionless mass matrix and $\omega^{*}$ is the dimensionless non-linear frequency .Using the tonsorial notation, one put:

$$
a_{i} k_{i r}^{*}+\frac{3}{2} a_{i} a_{j} a_{k} b_{i j k r}^{*}-a_{i} \omega^{* 2} m_{i r}^{*}=0 \quad \text { with } \quad r=1, \ldots, n
$$


The called the second formulation [17], it is an approximation which consists to separate the nonlinear term of equation (34) into a terms proportional to $a_{1}^{3}$ and terms proportional to $a_{1}^{2} \varepsilon_{i}$ and neglecting terms proportional to $a_{1} \varepsilon_{i} \varepsilon_{j}$ and terms proportional to $\varepsilon_{i} \varepsilon_{j} \varepsilon_{k}$ one can write:

$$
a_{i} a_{j} a_{k} b_{i j k r}=a_{1}^{3} b_{111 r}+a_{1}^{2} \varepsilon_{i} b_{11 i r} \quad r=1, \ldots, n
$$

After substituting and rearranging, equation (34) may be written in matrix form as:

$$
\left(\left[K_{r}^{*}\right]_{R}-\omega^{2}\left[M_{r}^{*}\right]_{R}\right)\left\{A_{r}\right\}_{R}+\frac{3}{2}\left[\alpha^{*}{ }_{r}\right]_{R}\left\{A_{r}\right\}_{R}=\left\{-\frac{3}{2} a_{r}^{3} b_{111 r}^{*}\right\}
$$

\section{RESULATS AND DISCUSSION}

In this present work, the free in-plane vibration of a FGM circular arch made with mixture of ceramic-metal material with uniform section and pinned at the both ends is studied based on multimode approach.

By assuming the material properties in table.1, the material volume fraction $\mathrm{p}$ dependence to the variation of the first four in-plane free vibration frequencies of FGM pinned-pinned circular arch are shown in Fig. 2 for radius $\mathrm{R}=60 \mathrm{~m}$, thicknesses $\mathrm{h}=0.8 \mathrm{~m}$ and an opening angle $\varphi_{0}=100^{\circ}$.

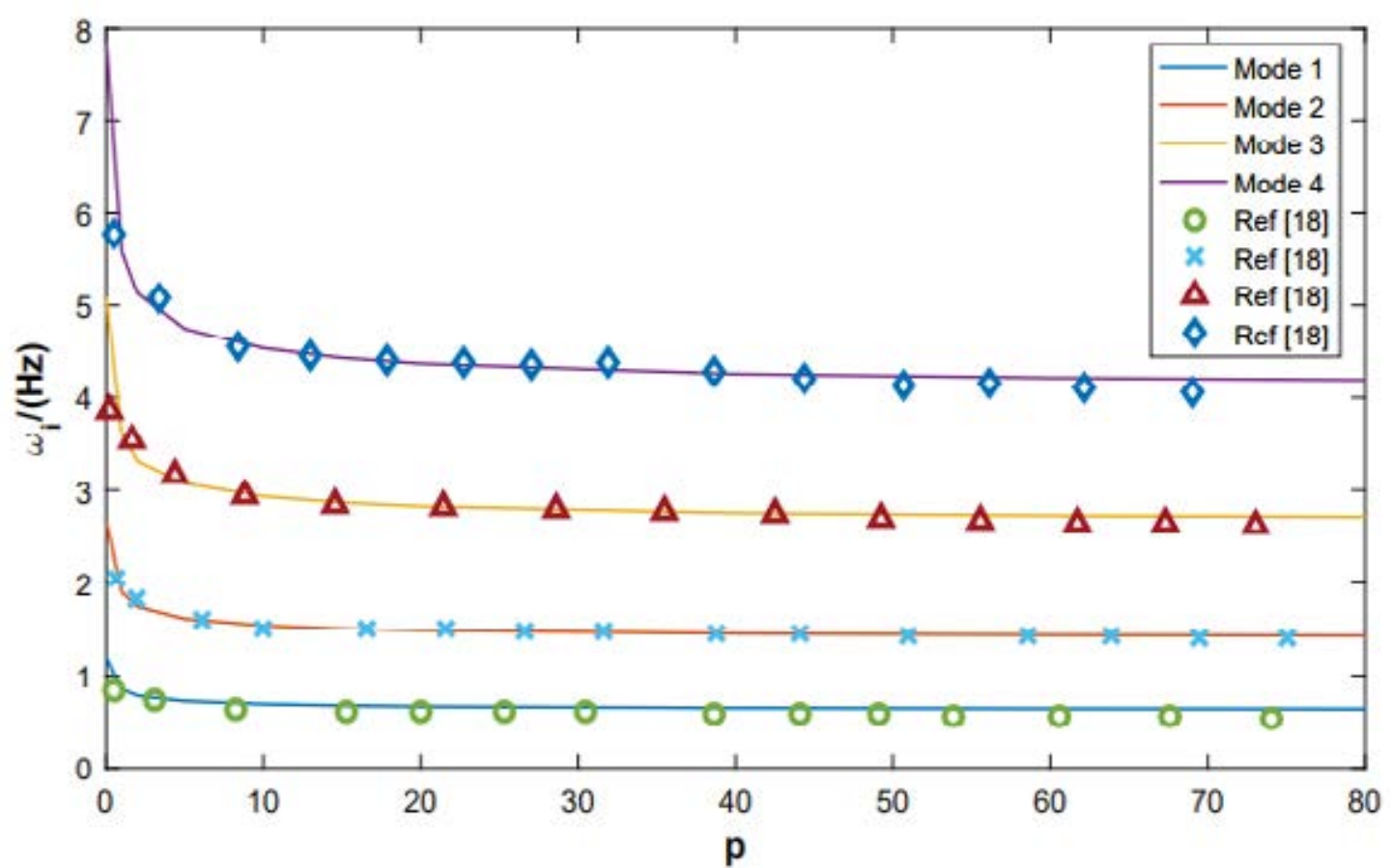

Fig. 2: The material volume fraction $p$ dependence to the variation of the first four in-plane free vibration frequencies of FGM circular arch [18].

The influence of the volume fraction index $\mathrm{p}$ on the dimensionless non-linear frequency ratio of pinned-pinened FGM circular arch are presented in Fig.4. 
The Fig. 2 shows a comparaison of the first four linear frequencies of the FGM pinned-pinned circular arch obtained in this present work with the results obtained in [18] as the curvature is constant. Where good agreement is obtained.

The effects of the opening angle of the FGM circular arch with a volume fraction index $\mathrm{p}=5$ and pinned-pinned ends on the dimensionless non-linear frequency ratios are shown in Fig. 4.

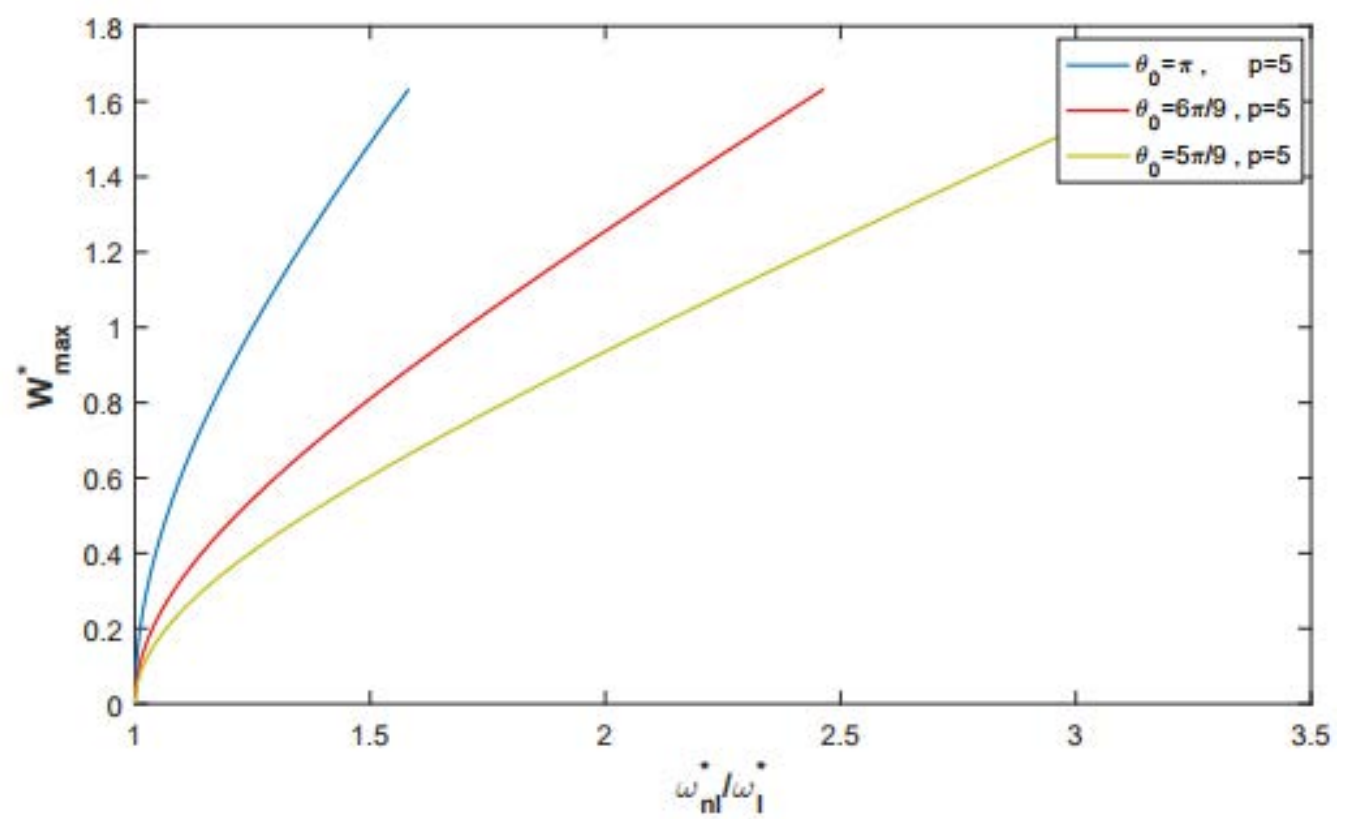

Fig. 3. Influence of the opening angle on the ratio of the dimensionless non-linear frequency ratios to the linear frequency of FGM circular arch beams with volume fraction $\mathrm{p}=5$.

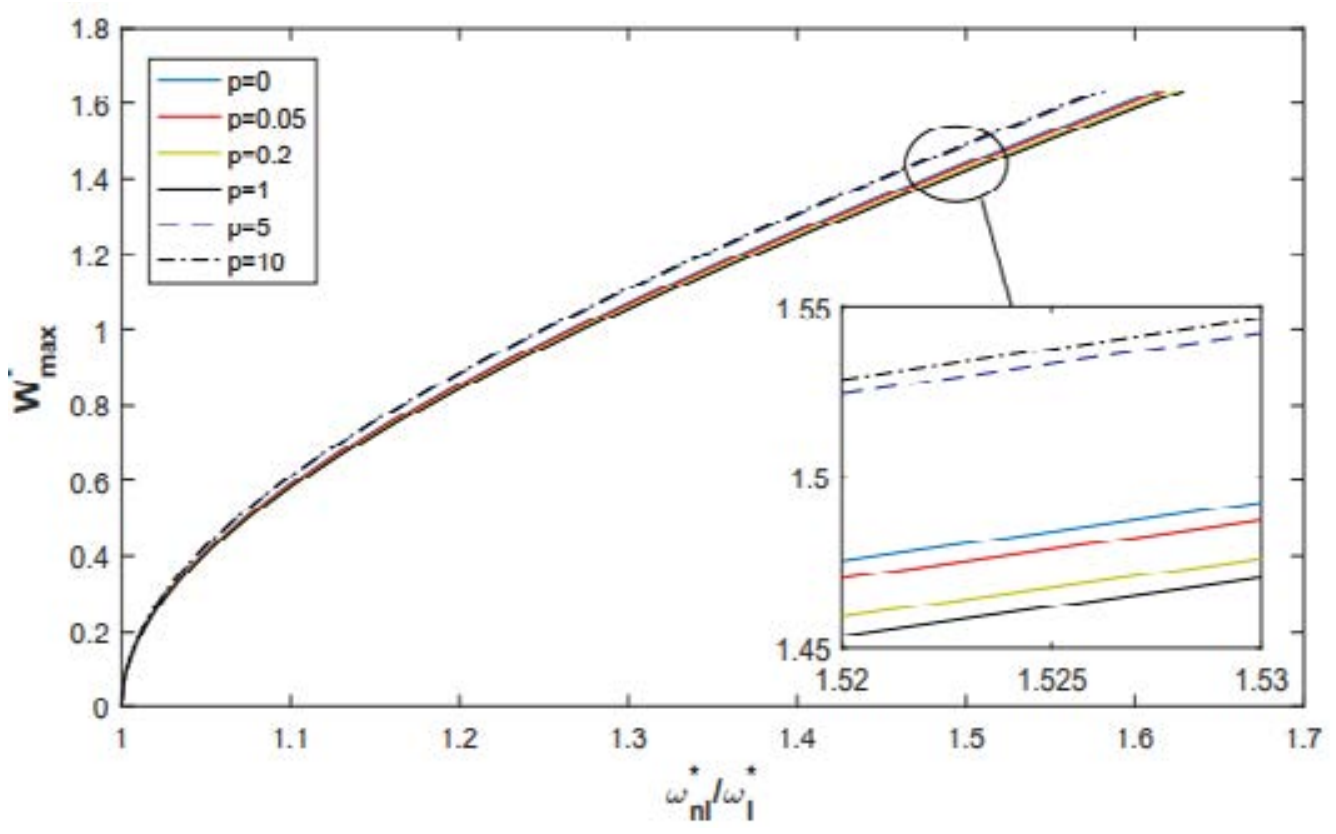

Fig. 4. Effect of The material volume fraction $\mathrm{p}$ on the frequency ratio.

The results in Fig. 4 show that the dimensionless non-linear frequency ratios of arch tend increase with increasing the opening angle increases, due to the increased rigidity. 
The influence of the volume fraction index $\mathrm{p}$ on the dimensionless non-linear frequency ratios of the FGM arch by considering the pinned-pinned boundary conditions can be clearly shown in Fig. 3.

A quantitative values of the dimensionless non-linear frequency ratios of the FGM circular arch, for various values of of the volume fraction index $\mathrm{p}$ and the non-dimensional amplitude of vibration are presented in Table 2. The results are compared with those obtained in [5].

Table 2: comparison of the dimensionless non-linear frequencies ratios for each value of the volume fraction index $\mathrm{p}$ with isotropic homogenous pinned-pinned circular arch [5].

\begin{tabular}{|c|c|c|c|c|c|c|c|c|}
\hline \multirow[t]{2}{*}{$W_{\max }^{*} / h$} & \multirow{2}{*}{$\begin{array}{c}\omega_{n l}^{*} / \omega_{l}^{*} \\
\operatorname{Ref}[5]\end{array}$} & \multicolumn{7}{|c|}{ Present $\omega_{n l}^{*} / \omega_{l}^{*}$} \\
\hline & & $\mathrm{p}=\mathbf{0 . 0}$ & $p=0.05$ & $\mathrm{p}=0.2$ & $p=1$ & $p=5$ & $p=10$ & $\mathbf{p}=\infty$ \\
\hline 0.08165 & 1.002004 & 1.002004 & 1.002017 & 1.002048 & 1.002064 & 1.001877 & 1.001867 & 1.002004 \\
\hline 0.46947 & 1.064259 & 1.064259 & 1.064694 & 1.065658 & 1.066144 & 1.60301 & 1.059994 & 1.064259 \\
\hline 0.857231 & 1.200968 & 1.200968 & 1.202254 & 1.205100 & 1.206531 & 1.189286 & 1.188324 & 1.200968 \\
\hline 1.24488 & 1.390340 & 1.390340 & 1.392682 & 1.397860 & 1.400463 & 1.368994 & 1.367231 & 1.390340 \\
\hline 1.63239 & 1.613943 & 1.613943 & 1.617412 & 1.625076 & 1.628927 & 1.582246 & 1.579621 & 1.613943 \\
\hline
\end{tabular}

Table 2 shows the dependence of the frequency ratio of the pinned FG circular arch on the amplitude of vibration for various values of the power law index $\mathrm{p}$. As may be seen in this figure, by increasing the values of the power law index in the range $[0,1]$, the frequency increases. For values higher than $p=1.0$, the frequency decreases when $p$ increases. This may be expected, since when the power index $\mathrm{p}=0.0$ or $\mathrm{p}=\infty$, the material becomes pure metallic or pure ceramic, respectively, and the non-dimensional frequency corresponds to the isotropic homogenous material case.

\section{CONCLUSIONS}

The geometrical non-linearity in free in-plane vibration of inextensible FGM circular arches. were analyzed based on the Euler Bernoulli theory and the Von Karman geometrical nonlinearity assumptions. For simplification, the complicating effects such as rotary inertia and shear deformation will be ignored. The naturals frequencies of arch were obtained iteratively using Newton-Raphson algorithm. The kinetic and total strain energy of the arch were reduced discretized then the problem was reduced into a set non-linear algebraic equations using Hamilton's principle energy and spectral analysis and solved numerically by an approximate explicit method the so-called second formulation. A numerical results were obtained to examine the effects of the volume fraction index on non-linear behavior of the FGM circular arch. 


\section{REFERENCES}

[1] H. Saffari, R. Tabatabaei, and S. H. Mansouri, 'Vibration Analysis of Circular Arch Element Using Curvature', Shock and Vibration, vol. 15, no. 5, pp. 481-492, 2008, doi: $10.1155 / 2008 / 149393$.

[2] P. A. A. Laura and P. L. V. de Irassar, 'A note on in-plane vibrations of arch-type structures of non-uniform cross-section: The case of linearly varying thickness', Journal of Sound and Vibration, vol. 124, no. 1, pp. 1-12, Jul. 1988, doi: 10.1016/S0022460X(88)81402-0.

[3] A. Babahammou and R. Benamar, 'Semi-Analytical Solution of In-Plane Vibrations of Circular Arches Carrying Added Point Masses', Procedia Manufacturing, vol. 44, pp. 465-472, Jan. 2020, doi: 10.1016/j.promfg.2020.02.269.

[4] A. Babahammou. B.Rhali, 'The Efficiency of the Rayleigh-Ritz Method Applied to InPlane Vibrations of Circular Arches Elastically Restrained against Rotation at the Two Ends', International Journal of Engineering Trends and Technology - IJETT, Accessed: May 30, 2021.

[5] O. Outassafte, A.Adri, Y.El khouddar, S.Rifai, B. Rhali, 'Geometrically Non-Linear Free In-Plane Vibration Of Circular Arch Elastically Restrained Against Rotation At The Two Ends', International Journal of Engineering Trends and Technology - IJETT, Accessed: Apr. 17, 2021. [Online]. Available: https://www.ijettjournal.org/archive/ijett-v69i3p215

[6] L. Boutahar, el bikri Khalid, and B. Rhali, 'Thermal Behavior Analysis at Large Free Vibration Amplitudes of Thin Annular FGM Plates with Porosities', Procedia Engineering, vol. 199, pp. 528-533, Dec. 2017, doi: 10.1016/j.proeng.2017.09.148.

[7] M. Chajdi, A. Ahmed, K. E. Bikri, and R. Benamar, 'Analysis of the associated stress distributions to the nonlinear forced vibrations of functionally graded multi-cracked beams', Diagnostyka, vol. 22, no. 1, pp. 101-112, Feb. 2021, doi: 10.29354/diag/133702.

[8] Y. E. khouddar, A. Adri, S. Rifai, and R. Benamar, 'Geometrically non-linear forced vibrations of Euler-Bernoulli laminated composite beams', J. Phys.: Conf. Ser., vol. 1896, no. 1, p. 012016, Apr. 2021, doi: 10.1088/1742-6596/1896/1/012016.

[9] M. Chajdi, E. B. Merrimi, and el bikri Khalid, 'Geometrically Non-Linear Free and Forced Vibration of Clamped-Clamped Functionally Graded Beam with Discontinuities.', Procedia Engineering, vol. 199, pp. 1870-1875, Dec. 2017, doi: 10.1016/j.proeng.2017.09.117.

[10] H. Fakhreddine, A. Adri, M. Chajdi, S. Rifai, and R. Benamar, 'Geometrically nonlinear forced vibrations of fully clamped multi-span beams carrying multiple masses and resting on a finite number of simple supports', J. Phys.: Conf. Ser., vol. 1264, p. 012021, Jul. 2019, doi: 10.1088/1742-6596/1264/1/012021.

[11] H. Babaei, Y. Kiani, and M. R. Eslami, 'Large amplitude free vibration analysis of shear deformable FGM shallow arches on nonlinear elastic foundation', Thin-Walled Structures, vol. 144, p. 106237, Nov. 2019, doi: 10.1016/j.tws.2019.106237.

[12] M. Javani, Y. Kiani, and M. R. Eslami, 'Free vibration of arbitrary thick FGM deep arches using unconstrained higher-order shear deformation theory', Thin-Walled Structures, vol. 136, pp. 258-266, Mar. 2019, doi: 10.1016/j.tws.2018.12.020.

[13] P. Malekzadeh, M. M. Atashi, and G. Karami, 'In-plane free vibration of functionally graded circular arches with temperature-dependent properties under thermal environment', Journal of Sound and Vibration, vol. 326, no. 3-5, pp. 837-851, Oct. 2009, doi: 10.1016/j.jsv.2009.05.016.

[14] M. Javani, Y. Kiani, and M. R. Eslami, 'Geometrically nonlinear rapid surface heating of temperature-dependent FGM arches', Aerospace Science and Technology, vol. 90, pp. 264-274, Jul. 2019, doi: 10.1016/j.ast.2019.04.049. 
[15] H. Asgari and M. Eslami, 'Nonlinear Thermal Buckling Analysis of FGM Shallow Arches Under Linear Temperature Gradient', ASME 2014 12th Biennial Conference on Engineering Systems Design and Analysis, ESDA 2014, vol. 1, Jul. 2014, doi: 10.1115/ESDA2014-20402.

[16] P. Chidamparam and A. W. Leissa, 'Vibrations of Planar Curved Beams, Rings, and Arches', Appl. Mech. Rev, vol. 46, no. 9, pp. 467-483, Sep. 1993, doi: 10.1115/1.3120374.

[17] M. El kadiri, R. Benamar, and R. G. White, 'IMPROVEMENT OF THE SEMIANALYTICAL METHOD, FOR DETERMINING THE GEOMETRICALLY NONLINEAR RESPONSE OF THIN STRAIGHT STRUCTURES. PART I: APPLICATION TO CLAMPED-CLAMPED AND SIMPLY SUPPORTED-CLAMPED BEAMS', Journal of Sound and Vibration, vol. 249, no. 2, pp. 263-305, Jan. 2002, doi: 10.1006/jsvi.2001.3808.

[18] W. Li and Z. Teng, 'In-plane free vibration analysis of FGM arches with variable curvature', Zhendong yu Chongji/Journal of Vibration and Shock, vol. 36, pp. 201-208, May 2017, doi: 10.13465/j.cnki.jvs.2017.09.030. 